\title{
Urgences
}

\section{L'autre e(s)t l'une. À propos d'une chanson de Clémence}

\section{André Gervais}

Numéro 26, décembre 1989

Des textes qui chantent

URI : https://id.erudit.org/iderudit/025553ar

DOI : https://doi.org/10.7202/025553ar

Aller au sommaire du numéro

Éditeur(s)

Urgences

ISSN

0226-9554 (imprimé)

1927-3924 (numérique)

Découvrir la revue

Citer cet article

Gervais, A. (1989). L'autre e(s)t l'une. À propos d'une chanson de Clémence.

Urgences, (26), 39-46. https://doi.org/10.7202/025553ar d'utilisation que vous pouvez consulter en ligne.

https://apropos.erudit.org/fr/usagers/politique-dutilisation/ 


\section{L'autre e(s)t l'une À propos d'une chanson de Clémence}

\section{André Gervais}

L'amante et l'épouse a d'abord été une chanson accompagnant un sketch dans la revue intitulée Les girls de Clémence DesRochers, mise en musique par François Cousineau et jouée à Montréal, au Patriote à Clémence, de mai à juillet $1969^{1}$, puis, les années passant, le public se renouvelant et un répertoire se développant, s'épurant, se reconfigurant, une chanson tout court, recontextualisée en quelque sorte ${ }^{2}$ et actuellement enregistrée deux fois, la

1 Cette revue, qui n'a pas fait alors l'objet d'un disque, a toutefois été partiellement publiée dans La grosse tête, coll. a Mon pays mes chansons", Montréal, Leméac, 1973, p. 33-69. Les róles étaient tenus par Paule Bayard, Diane Dufresne - qui rencontre François Cousineau à cette occasion et fera par la suite, à partir de 1972, avec des chansons écrites par Luc Plamondon et François Cousineau, entre autres, la carrière que l'on sait -, Louise Latraverse, Chantal Renaud et par l'auteur. Dans cotte revue, me dit (le 22 aoot 1989) Hélène Pedneault qui, au moment où paraît cette brève analyse, vient tout juste de publier Notre Clémence. Tout l'humour du vrai monde (coll. « Paroles d'ici". Montréal, Éd. de l'Homme, 1989), la chanson est interprétée par Diane Dufresne (épouse) et Louise Latraverse (amante). Est-ce un hasard si « anniversaire ", synonyme de a fête" (v. 21 et 23), mot important du texte comme on le verra, peut se lire dans les prénom et nom de ses premières interprètes ainsi que dans le nom de l'auteur: * anni " (Dlane) / « vers " (Latraverse)/ * aire " (DesRochers) ? 2 De la méme façon, La chaloupe Verchères fait partie, en un premier temps, de C'est pas une revue, c't'un show, revue présentée en 1971, et partiellement publièe elle aussi dans La grosse tête, p. 113-136 (la chanson est aux p. 124126). Chaque strophe, chantée, y est brièvement entrecoupee par la * voix du père ", parlant et représentant le père référentiel - le poète Alfred DesRochers -, joué par le jeune chanteur Ovila B. Blais; la chanson se termine par la voix de la chanteuse - Clémence, sa fille - qui, parlant, réveille le père endormi, et par la “ voix du père". En un second temps, la chanson tout court - sans les voix parlées du père et de la fille, donc - est enregistrée dès 1971 sur un 45 tours, puis en 1975 sur un long-jeu (voir note suivante). En un troisième temps, à l'occasion du spectacle Mon dernier show, enregistré sur disque en avril 1977, un fragment de cette chanson - les trois premieres strophes - est lié a des fragments de trois autres chansons pour faire une nouvelle chanson qui n'est ni un pot-pourri (de succès), ni un mixage (de bandes d'époques diverses), mais, littéralement, servi par une voix très juste et retenue, et unifié par des arrangements d'une sobriété et d'une efficacité remarquables, un ensemble d' « Hommages " - c'est le titre - au père et à la mère. Dans l'ordre: L'homme de ma vie (1965), La chaloupe Vercheres (1971). Enquête (entre 1960 et 1962) et Avec les mots d'Alfred (1976). II va sans dire que je tiens cette chanson deux 
40

première par Clémence DesRochers (épouse) et Marie-Michèle Desrosiers (amante), la seconde par Renée Claude (amante) et Louise Forestier (épouse) ${ }^{3}$. Le texte de la chanson a été publié, sauf erreur, trois fois : la première et la troisième dans un livre de l'auteur ${ }^{4}$, la seconde sur une feuille accompagnant le premier enregistrement. Le voici:

Ça fait trois jours que j’te vois pas

Mon mari, mon époux, mon homme.

Tu viens trois jours et tu t'en vas

Mon ami, mon amant, mon homme.

5 Une autre femme a fait de toi

Son ami, son amant, son homme.

Une autre femme a fait de toi

Son mari, son époux, son homme.

Tu ne peux pas te passer d'elle

10 Tu dis que c'est le grand amour.

Tu ne peux pas vivre sans elle

Tu dis que tu l'aimes toujours.

Je l'imagine grande et belle

J'ai peur que tu me quitt's un jour.

Je l'imagine forte et belle

J'ai peur que tu me quitt's un jour.

Quand tu n'es pas là c'est la défaite

Les enfants s'inquiètent de toi.

Quand tu n'es pas là je suis défaite

Tu ne veux pas d'enfant de moi.

fois composée pour la plus importante de Clémence: investissement autobiographique, choix et agencement des fragments, plasticité des musiques (dans l'ordre: de Pierre F. Brault, de Gaston Brisson, de Jean-Marie Cloutier et de Marc Larochelle), entre autres.

3 Clémence DesRochers (ici CD), Comme un miroir, Franco Disque Inc., FR793, enregistré en aout 1975. Sur ce disque, L'amante et l'épouse cotoie, par exemple, les premières chansons écrites avec et accompagnées par les frères Larochelle: Full day of mélancolie et Le monde aime mieux Mireille Mathieu. Renée Claude (ici RC), L'enamour le désamour, London, LFS-9019, enregistré en août 1976. Mais cette chanson, ici repiquée, est déjà sur un 45 tours enregistré, lui aussi, en 1975 (London, LF-1061 / JPB-122).

4 Clémence DesRochers, La grosse téte, p. 60-611; Le monde aime mieux..., préface de Marc Favreau, illustrations de Jean Daigle, Montréal, Éd. de l'Homme, 1977, p. 65-68. J'ajoute que dans son livre (voir note 1) Hélène Pedneault publie aussi le texte (p. 371-372), mais avec encore une autre coda. $A$ n'en pas douter, le nooud de cette chanson. 
Ils t'ont préparé une fête

Je sais que tu nous reviendras.

Tu oublieras que c'est ma fête

Je n'sais jamais si tu viendras.

(version disque et version livres - version non enregistrée)

25 Mon ami, mon amant, mon homme

Mon mari, mon époux, mon homme.

(enregistrement $C D$ )

25 Mon mari, mon homme, mon époux, mon homme

Mon ami, mon homme, mon amant, mon homme.

(enregistrement RC)

25 Mon ami, mon amant, mon homme

Mon mari, mon époux, mon homme

Mon ami,

Mon mari, mon époux, mon homme

Mon mari,

3o Mon amant,

Mon époux, Mon homme.

Il n'est pas inutile de faire ici les deux remarques suivantes ${ }^{5}$ :

- la disposition «épouse „ à la marge/ "amante * en retrait est commune à la version disque et à la version livres, la version disque (pour l'enregistrement $C D$ ) précisant même la chose par l'ajout de deux petites flèches;

- la version disque s'arrête au v. 26 dans l'enregistrement $\mathrm{CD}$, au v. 32 - que je dispose à mi-chemin exactement des autres parce que les interprètes chantent alors en même temps - dans l'enregistrement RC (que je considérerai, essentiellement).

Cela n'est pas sans conséquences, comme on le verra.

La chanson est faite de trois parties regroupant chacune deux strophes (v. 1-8, 9-16, 17-24) - chaque strophe regroupant deux distiques - et d'une coda (v. 25 et s.). Trois strophes (I, II et $V$ ) regroupent des vers de huit et neuf

5 Deux différences entre ce qui est écrit et ce qui est chante : au v. 15, aforte " (version La grosse tête, version disque ainsi qu'enregistrements CD et RD) plutót que a grande " (version Le monde aime mieux...); aux v. 14 et 16, "quilt's " (enregistrements) plutôt que “ quittes " (versions). 
syllabes: "Mon mari, mon époux, mon homme» (v. 2), par exemple, a huit syllabes si l'on ne considère que le texte, mais en a neuf (trois fois trois) si l'on considère la musique qui l'accompagne. Les trois autres strophes (III, IV et VI) regroupent des vers de huit syllabes. Chaque distique a ses terminaisons masculine et féminine (ou l'inverse): il faut donc bien deux distiques - l'un laissé à l'épouse, l'autre à l'amante pour que ces fins de vers riment entre elles. Les première et dernière parties ont les mêmes rimes masculines, mais des rimes féminines particulières, façon de boucler la situation d'une part en faisant rimer deux fois deux mots de trois lettres ("pas"/ "vas", "toi " " moi ") ou de trois et deux syllabes ( reviendras »/ « viendras »), d'autre part en laissant entendre, dans les sections rimantes, qu'entre a ou a («-as»/ «-oi »), tour ou retour, il n'y a(urait) pas à choisir.

Musicalement, chaque partie joue sur deux mélodies : l'une, associée à la première strophe, chantée plus en douceur - bien qu'au v. 5, Louise Forestier (épouse) insiste, en tant qu'épouse, avec le phrasé qui lui est caractéristique, sur les « mm» ("aime"?) d'*Une autre femme» -, débutant dans la transcription publiée sur un accord de do mineur (les trois premières notes sont $m i$ bémol, $f a$ et $\mathrm{sol}$ ); l'autre, associée à la deuxième strophe, chantée de façon plus accentuée, sur un accord de sol mineur (les trois premières notes sont sol, la, si bémol). Autant il y a la voix douce, feutrée et neutre - sereine? - de Renée Claude, autant il y a la voix (discrètement) énergique - « forte » (v. 15)? - et mélancolique de Louise Forestier.

Mais où commence une chanson? Aux premières mesures jouées, aux premières paroles chantées, au titre lu sur la pochette du disque ou dans le programme du spectacle, ou encore lorsque les paroles sont à peu près écrites avant la musique (comme c'est le plus souvent le cas chez Clémence DesRochers) ou lorsqu'elles parviennent à se mettre en place sur celle-ci (et celle-ci sur elles)? Et où finit une chanson, quelle version peut être la version (du point de vue des paroles, du point de vue des arrangements et de l'interprétation) qui pourrait être dite définitive? On s'en doute, cette chanson a d'abord le mérite de pointer quelques-unes de ces questions en proposant ceci: une chanson peut commencer dans une apparente contradiction (l'wamante - deux ou trois syllabes - d'abord dans le titre, l'« épousen - également deux ou trois syllabes - d'abord dans le chant) en 
dirigeant les deux voix - ou voies - vers un même objet absent mais partout présent ( mon homme") et en alternant les déclarations, et une chanson peut finir, du moins dans l'enregistrement RC, sur ces deux mots et leurs trois syllabes chantés par les deux voix en même temps. Ce qui est séparé par la marge est uni par la strophe; ce qui se fait voix distincte (de l'épouse d'abord, première quittée, ensuite de l'amante) est uni par le titre (L'amante et l'épouse) où, comme on le voit, la première place est accordée à l'amante; ce qui est, dans les faits, opposition (l'«épouse» versus l'«amante») devient, dans le texte, équivalence (l'amante e(s)t l'épouse).

Si l'on exclut la coda pour le moment, le texte, en effet, présente trois moments (qui recoupent les trois parties) et deux situations: l'absence (du même homme), la présence (de l'autre femme). Dans la première partie, absence et présence; dans la seconde, présence; dans la troisième, absence (compliquée par l'ajout d'enfants). Â chaque moment, elle - amante ou épouse parle à l'homme et, sauf au dernier moment, de l'autre: le dernier moment, celui de la dramatisation, est en effet réservé non plus à l'échange de qualités - chaque femme allant même jusqu'à formuler ce que l'homme lui dit de l'autre, jusqu'à imaginer l'autre -, mais au repliement là sur la famille, ici sur le couple.

Mais ce qui fait la liaison d'un moment à l'autre est bien ceci, côté épouse: « Ça fait trois jours que j’te vois pas» (v. 1), "Une autre femme a fait de toi" (v. 5), "Tu ne peux pas te passer d'elle" (v. 9), "Je l'imagine grande et belle" (v. 13), "Quand tu n'es pas là c'est la défaite / Les enfants s'inquiètent de toi (v. 17-18), «Ils t'ont préparé une fête / Je sais que tu nous reviendras" (v. 21-22). Tout y est: de l'absence à la nonabsence, l'hypogramme caché au début du premier vers de la première strophe devient ouvertement, dans le premier vers de la dernière strophe, le mot de la fin ( «fait $\mathrm{t}$-»/ «fête»), avec, entre ces extrêmes dont l'issue semble volontairement suspendue, le surgissement d'un "de" qui devient progressivement un «dè" puis un « dé» - comme, exactement, le «Des» de DesRochers et qui pose l'équivalence "défaite" / "fête" 6 . Côté amante,

6 "Défaite" est ici le contraire de "fête", comme "shakos " le pluriel de "chacal " (chez le Flaubert du Dictionnaire des idées reçues), ou " rebelle "le renforcement de a belle " (chez le Duguay de l'aphorisme préinfoniaque, 1968 pour être précis: a La poésie n'est pas que belle elle est rebelle "). Dans les trois cas, le calembour et une régle de la grammaire française forment le point d'ancrage. 
44

l'élaboration est moindre: son discours, qui ne fait en quelque sorte, donnant sa version, que répondre au discours de l'épouse, en devient même, dans la coda de l'enregistrement RC (v. 28-31), l'écho absolument autre. Ainsi, à "Une autre femme a fait de toi / Son ami, son amant, son homme" (v. 5-6) répond "Une autre femme a fait de toi / Son mari, son époux, son homme " (v. 7-8): c'est la dernière fois avant la coda où les deux groupes de trois noms sont prononcés - la ressemblance entre "ami », "amant " et " homme " est déjà plus grande — et la seule fois où ils sont échangés par le biais de l'adjectif possessif de la troisième personne. Mais il y a aussi à entendre, entre *Une autre femme a fait de toi» et "Tu oublieras que c'est ma fête" (v. 23), le mot même autour duquel tout se noue - « Quand tu n'es pas là je suis défaite" (v. 19) - , repris en sourdine dans "Tu ne veux pas d'enfant de moi » (v. 20).

Et «l'épouse» et «l'amante», épinglées par l'article défini, s'adressent à "mon homme» en lui disant « tu », mais le répondant de ce dialogue on ne peut plus ordinaire - de * je " à " tu * - est maintenant absent, les v. 10 et 12 au moins impliquant un dialogue, voire une discussion passée. Comme si les deux voix, d'entremêler désormais leur monologue, puisque l'homme s'est tu, se parlaient et que c'était ça, plutôt, le dialogue. Cette théâtralisation du triangle amoureux — * Ça fait trois jours » dit l'une, "Tu viens trois jours " dit l'autre - se joue, de fait, à deux, en l'absence intime de l'intimé, nécessaire point aveugle 7 .

De *c'est la défaite ", dit l'une, à «je suis défaite ", dit l'autre, il y a le passage du constat objectif (c'est la déroute, la bataille est perdue) à l'état subjectif (je suis abattue); et malgré la certitude, à la fin, du * Je sais que tu nous reviendras " (v. 22) de la première - la certitude quelque peu béate du retour au bercail, à la stabilité de la cellule familiale - et l'incertitude du *Je n'sais jamais si tu viendras" (v. 24) de la seconde - la difficile incertitude qu'engendre tout programme non établi, voire non établissable - la possibilité de voir la balance finalement pencher est bien suspendue. Avec des intensités variables, "l'épouse » est «l'amante», participant toutes deux, hypogrammatiquement, de "Clémence *, au même titre que, comme il a été précédemment suggéré,

7 Cette façon de jouer la (co)présence en l'appuyant sur l'absence et sur une illusion de temporalité isochrone est, on le sait, l'une des caractéristiques de la piece de Michel Tremblay, $\dot{A}$ toi, pour toujours, ta Marie-Lou, jouee en 1971. 
"défaite " de «DesRochers". C'est dire que cette chanson, comme quelques autres textes, spécifiquement «littéraires", serait signée, et bien signée, de l'intérieur: "mon homme", prononcé par l'une et par l'autre, peut donc être aussi, en quelque sorte, «mon nom» 8 .

"Tu ne peux pas te passer d'elle» (v. 9), «Quand tu n'es pas là c'est la défaite" (v. 17), «Ms t'ont préparé une fête» (v. 21) dit l'épouse. Ces trois vers sont les seuls où une syllabe se répétant en encadre une autre. Dans le premier, "papa» est coupé, voire déchiré, dans le troisième, "pa» est réintégré, voire réparé après avoir été, dans le deuxième, exclus de la répétition: le "te", pronom réfléchi, est donc bien la dernière syllabe de "défaite " (associée à l'absence) et de "fête" ('’absence étant étayée, dans la dernière partie, par la présence d'enfants: « mari *, «époux ", mais aussi «papa»). Si «mari ", assonant avec « ami », propose déjà une certaine ressemblance entre les femmes et le statut de leur homme, "époux", synonyme mou de "mari", se distingue radicalement d' «amant», synonyme dur d'«ami \%. Que dire alors de la quatrième strophe (v. 13-16) où il n'y a qu'un seul mot de différence entre le dit de l'épouse à propos de l'autre et celui de l'autre à propos de l'épouse: "grande" (physiquement) doit être l'amante, « forte (psychologiquement) l'épouse, chacune leur air ( $r$ r) et leur part de lanneau ("an»/ "on), de l'alliance avec leur homme. Puis, implicite depuis la seconde partie, arrive "papa", mot fait non d'une assonance, mais du redoublement de sa syllabe. Le décalage exact - de "pas/te/pas/ser" à "là/ c'est/la puis de * pas/te/pas/ser * à «(p)ré/pa/ré» - met en scène non seulement du biographique (un "passén) associé au rôle de père, mais de la méconnaissance (un "sait pas «), de la part de la mère, face au caractère indispensable de l'amante : c'est plutôt nous, écouteurs de la chanson, qui pouvons mesurer, par le rapport constant des distiques dans les strophes, l'ampleur du "double-bind", ampleur d'autant plus perceptible que toutes les

8 Sans perdre de vue cec: a mon homme ", si l'on glisse - lapsus - d'une chanson à l'autre, c'est L'homme de ma vie, c'est (celui qui a) le (nom du) pere. Mais je donne un seul autre exemple: les deux derniers vers de l'Hymne au printemps (1949) de Félix Leclerc disent bien à la fois * Près du ruisseau sont alignées les fées / Et les crapauds chantent la liberté " et, dans la non-linéarité

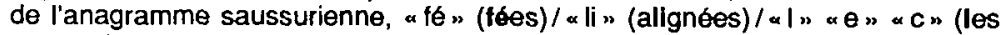
crapauds chantent) $/ \propto \mid$ " * or " (liberté). Comme quoi, si l'on ajoute $L \theta$ roi heureux (1949 également), l'onomastique est particulièrement sollicitante en cette année qui précède son départ pour la France. 
déclarations - constatations, nomination, argumentation, confidences - passent par des voix - et des voies - qui ne sont pas hargneuses, qui sont presque sereines. Il y a quelque chose du ton et de la situation de Jules et Jim dans L'amante et l'épouse ${ }^{9}$.

Si l'on considère maintenant la coda - les v. 25-32 dans l'enregistrement $\mathrm{RC}$, le dernier venu, je le rappelle -, il se passe une chose étonnante: Louise Forestier (l'épouse) reprend au v. 25 le v. 4 (chanté alors par l'amante) et Renée Claude ('amante) au v. 26 le v. 2 (chanté alors par l'épouse). Cette possibilité, qui est déjà celle de la version disque et de la version livres, actualise l'interchangeabilité des rôles et des pôles: le rapport épouse / époux est bien l'équivalent du rapport amante/amant. Louise Forestier redevenant, au v. 27, l'épouse, Renée Claude peut être une dernière fois l'amante (v. 28 et 30 ) avec Louise Forestier, en écho - mais l'écho est toujours second -, l'épouse (v. 29 et 31). Toujours le deux ("Tu ne peux pas te passer d'elle" aura dit l'épouse) et le trois ( Une autre femme a fait de toi » aurontelles dit). Le v. 32 ( Mon homme $)$ ), enfin, chanté par l'amante et l'épouse ensemble, est bien la charnière par laquelle tout aura été noué. Les décisions qui ont mené à l'élaboration de cette coda me semblent particulièrement habiles: ne permettent-elles pas à Louise Forestier - pour cette chanson, l'autre, invitée (et complice) de Renée Claude - de toujours être la première énonciatrice, peu importe le rôle qu'elle y joue ${ }^{10}$ ?

9 Je parle du film de François Truffaut (1962), mais aussi du roman d'HenriPierre Roché (1953) dont il est l'adaptation. Sans oublier, dans le film, la chanson intitulée Le tourbillon (paroles et musique de Cyrus Bassiak, voix de Jeanne Moreau), juste mise en abyme de son sujet. Jules et Jim, très amis, aiment Catherine: Jules et Catherine se marient, il a d'elle une petite fille; une fois Jules parti à la guerre - cela se passe dans les années 1910 - puis revenu, Jim devient l'amant de Catherine, sans que jamais son amitie pour Jules n'ait diminué. À la fin, Catherine et Jim se noient, sous les yeux de Jules. a J'ai trouvé le livre merveilleux et j'ai été frappé à la fois par le caractère scabreux des situations et la pureté de l'ensemble. [...] C'est [...] une histoire sur l'amour, avec cette idée que, le couple n'étant pas toujours une notion réussie, satisfaisante, il semble légitime de chercher une morale différente, d'autres modes de vie, bien que tous ces arrangements soient voués à l'échec " dira Truffaut dans une interview accompagnant la sortie du film (Jules et Jim, découpage intégral, coll. « Points ", F 6, Paris, Seuil / Avant-Scène, 1962 et 1971, p. 9 et 10). J et J/L' et l'.

10 Sur ce microsillon intitulé, je le rappelle, L'enamour le désamour, Renée Claude interprète aussi Faut que j'me pousse (paroles de Pierre Harel, musique de Pierre Harel et Gerry Boulet, 1971). Le jeu en-/dés-, fortement mis en scène dans celui de l'amante / épouse, ne l'est pas moins dans cette version inversée (" Téminisée ") de l'une des chansons les plus dures du groupe Offenbach. 